\title{
Intracranial hematoma in a patient with AIDS
}
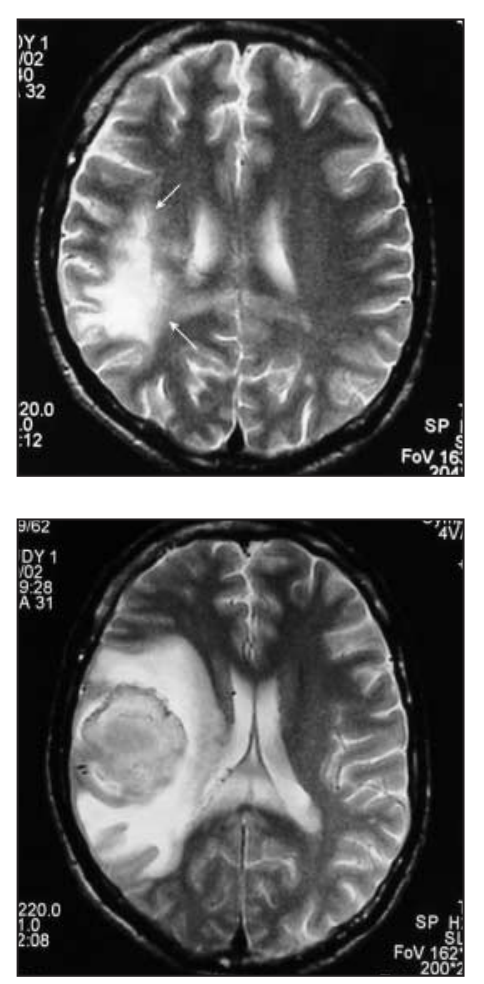

41-year-old man with HIV infection and chronic hepatitis $\mathrm{C}$ was admitted to hospital with a 4-week history of increasing cognitive decline and left hemiparesis. He was a former intravenous drug user. His HIV infection had been diagnosed and active antiretroviral therapy (HAART) started 4 years earlier. One year before admission, his CD4 cell count was 116 (normally $700-1100) \times 10^{6} / \mathrm{L}$, but he was healthy. At that time he stopped the HAART and was lost to follow-up.

On admission, the patient was afebrile and alert but was disoriented and had left hemiparesis and facial weakness, with gaze preference to the contralateral side. Cranial MRI revealed a large T2-weighted hyperintense lesion in the white matter of the right parietotemporal region, without mass effect (Fig. 1 , arrows), which suggested progressive multifocal leukoencephalopathy (PML). Blood tests showed elevated aminotransferase levels and a CD4 count of $95 \times 10^{6} / \mathrm{L}$. The prothrombin and partial thromboplastin times were normal, as were results of cerebrospinal fluid analysis. The polymerase chain reaction was positive for $\mathrm{JC}$ virus (JCV) DNA in cerebrospinal fluid.

A presumptive diagnosis of PML was made. HAART was restarted and the patient's neurologic symptoms improved over the following weeks. However, on day 25 , he became confused and experienced headache, vomiting, flaccid left hemiplegia and seizures. A second cranial MRI showed a large hemorrhage in the right parietal lobe, within the area of PML, with surrounding edema and mass effect (Fig. 2). Anticonvulsant therapy was started along with aggressive treatment to decrease intracranial pressure. Unfortunately, the patient's condition deteriorated, and he died.

At autopsy, a large (4-cm) hemorrhagic mass in the right parietal lobe was associated with extensive demyelinated lesions in the subcortical white matter of the right parietotemporal region. Microscopic examination revealed the characteristic pathological features of PML, and there were no signs of cerebral vasculitis, lymphoma or toxoplasmosis.

PML is a demyelinating disease of the central nervous system caused by JCV, a polyomavirus that induces a lytic infection of oligodendrocytes. Asymptomatic JCV infection is common in the general population $(80 \%-$ $90 \%$ of young adults have $\mathrm{IgG}$ antibodies against JCV), but PML is typically observed in immunocompromised people, affecting an estimated $5 \%$ of patients with AIDS. ${ }^{1}$ PML lesions consist of multiple coalescing areas of demyelination in the white matter of the cerebral hemispheres, brain stem, cerebellum and, rarely, spinal cord.

Clinically, PML is characterized by progressive neurologic deficits, predominantly mono- or hemiparesis, gait and speech disorders, visual loss and cognitive impairtment. Death occurs a median of 6 months after diagnosis. ${ }^{1}$ HAART has improved survival for some patients with PML (1year probability of survival has increased from $9 \%^{1}$ to about $50 \%^{2}$ ), although mortality during the first 3 months continues to be high $(30 \%-40 \%){ }^{2}$

Intracerebral hemorrhage is a rare complication of AIDS and is often due to hemophilia, thrombocytopenia, primary cerebral lymphoma, metastatic Kaposi's sarcoma and cerebral toxoplasmosis, none of which was present in our patient. Focal hemorrhage in PML lesions has rarely been reported. In our patient, a direct effect of JCV infection on cerebral vessels, associated with inflammatory changes, provides a possible explanation for the hemorrhage, as has been suggested for cerebral toxoplasmosis and other central nervous system infections.

\section{Eduardo Canalejo Castrillero Carmen Carratalá Blasco \\ Department of Internal Medicine Manacor Hospital Foundation \\ Manacor (Mallorca), Spain}

\section{References}

1. Berger JR, Pall L, Lanska D, Whiteman M. Progressive multifocal leukoencephalopathy in patients with HIV infection. 7 Neuroviol 1998;4:59-68.

2. Antinori A, Cingolani A, Lorenzini P, Giancola ML, Uccella I, Bossolasco $\mathrm{S}$, et al; Italian Registry Investigative Neuro AIDS Study Group. Clinical epidemiology and survival of progressive multifocal leukoencephalopathy in the era of highly active antiretroviral therapy: data from the Italian Registry Investigative Neuro AIDS (IRINA). 7 Neurovirol 2003;9(Suppl 1):47-53. 